\title{
A new interaction between Abi-1 and $\beta P I X$ involved in PDGF-activated actin cytoskeleton reorganisation
}

\author{
Fanny Campa ${ }^{1,2}$, Nikolaus Machuy ${ }^{1}$, Alexander Klein $^{1}$, Thomas Rudel $^{1}$ \\ ${ }^{1}$ Max Planck Institute for Infection Biology, Department of Molecular Biology, Campus Charité Mitte, Charitéplatz 1, Berlin 10 117, \\ Germany; ${ }^{2}$ Laboratory of Cellular Oncology, Center for Cancer Research, National Cancer Institute, Bethesda, MD 20892, USA
}

Members of the Rho family of GTPases are key regulators of the actin cytoskeleton. In particular, activated Rac1 stimulates membrane dorsal ruffle formation in response to platelet-derived growth factor (PDGF). Abl-interactor (Abi)1 and $\beta$ PIX, a guanine nucleotide exchange factor for Rac1, localise at these Rac1-induced actin structures and play important roles in the induction of membrane dorsal ruffling in response to PDGF in fibroblasts. Here, we demonstrate a novel interaction between Abi-1 and $\beta$ PIX using the yeast two-hybrid system, in vitro pull-down assays, and in vivo co-immunoprecipitation experiments. In vitro, the C-terminal fragment of $\beta \mathrm{PIX}$ interacted with Abi-1, while in vivo the $\mathrm{N}$-terminal fragment of $\beta$ PIX interacted with Abi-1. The biological function of this interaction was investigated in mouse fibroblasts in response to PDGF stimulation. Abi-1 and $\beta$ PIX co-localised in the cytoplasm and to membrane dorsal ruffles after PDGF treatment. We show that the co-expression of Abi-1 and truncated forms of $\beta$ PIX in mouse fibroblasts blocked PDGF-induced membrane dorsal ruffles. Together, these results show that the interaction between Abi-1 and $\beta$ PIX is involved in the formation of growth factor-induced membrane dorsal ruffles.

Cell Research (2006) 16:759-770. doi: 10.1038/sj.cr.7310091; published online 29 Aug 2006

Keywords: $\beta$ PIX, Abi-1, PDGF, membrane dorsal ruffling, cytoskeleton

\section{Introduction}

Regulation of the actin cytoskeleton plays a crucial role in many cellular functions such as cell shape change, cell motility, cell adhesion, and cytokinesis [1]. Small GTPases of the Rho family are key regulators in the transduction of signals from membrane receptors to the actin cytoskeleton [2]. Their activation requires the exchange of GDP for GTP, triggered by the action of guanine nucleotide exchange factors (GEFs) in response to extracellular signals like the platelet-derived growth factor (PDGF) [3]. Active RhoA proteins regulate stress fibre formation and the assembly of focal adhesions [4], while active Rac1 regulates membrane

Correspondence: Thomas Rudel

Tel: +49-30-28460-415; Fax: +49-30-28460-401

E-mail: rudel@mpiib-berlin.mpg.de

Received 24 Feb 2006; revised 30 Jul 2006; accepted 31 Jul 2006; published online 29 Aug 2006 ruffle, lamellipodia formation, and focal complexes [5], and active $\mathrm{Cdc} 42$ regulates filopodia formation [6].

The Abl-interactor (Abi) proteins were shown to localise to sites of de novo actin polymerisation at the tips of lamellipodia and filopodia in migrating cells [7]. The Abi protein family [8] is an emerging class of cytoplasmic molecular adaptors with an Src homology 3 (SH3) domain, similar to Grb2 or Nck [9]. The human Abi-1 protein was initially identified in a yeast two-hybrid screen as interaction partner of Eps8, a substrate for growth factor receptor tyrosine kinases [10]. Abi-1 contains several PxxP (proline-rich region) motifs that bind to the $\mathrm{SH} 3$ domain of c-Abl [11], Grb4 [12], spectrin [13], and synaptojanin [14]. Abi-1 also contains a PxxDY (unconventional proline-rich region) motif, which has been shown to bind to Eps8 [15]. Several sequences rich in proline, glutamic acid, serine, and threonine (PEST sequences), which function as signals for rapid intracellular proteolysis, are also present [16]. The SH3 domain at the C-terminus of Abi-1 interacts with 
the proline-rich domains of c-Abl $[11,17,18]$ and Sos-1 [19]. Abi-1 is implicated in the regulation of c-Abl, which is involved in membrane dorsal ruffling in PDGF-treated fibroblasts [20].

Abi-1 has been shown to participate in the formation of membrane dorsal ruffles in response to PDGF [21]. One such mechanism has been elucidated. The PDGF signal is transmitted from Ras to Rac1 through a trimeric complex containing Abi-1, Eps8, and Sos-1, in which Abi-1 holds Eps8 and Sos-1 together [19, 21, 22]. More specifically, Abi-1 recruits the phosphatidylinositol 3-kinase (PI3-K), through a direct interaction with the $\mathrm{p} 85$ subunit of PI3-K, to a multimolecular signalling complex including Eps8 and Sos- 1 . The recruitment of PI3-K and its catalytic product phosphatidylinositol 3, 4, 5 phosphates (PIP3), which regulates the activity of the GEF protein Sos-1, are indispensable for the activation of Rac1 and actin cytoskeleton remodelling in response to PDGF [23]. Thus, it can be easily imagined that as Abi- 1 is implicated in the regulation of PDGF-induced membrane dorsal ruffling, other GEF proteins besides Sos- 1 could also be involved in this process.

The human $\beta$ PIX protein, designated as a $\mathrm{p} 21$-activated kinase (PAK)-Interacting eXchange protein, belongs to the family of GEFs [24, 25]. $\beta$ PIX, a GEF for Rac1, contains an SH3 domain followed by a Dbl homology (DH) domain in tandem with a pleckstrin homology $(\mathrm{PH})$ domain (Figure 1). The DH domain is directly responsible for the guanine nucleotide exchange activity and activation of the small Rho GTPases [26, 27]. However, the DH and PH domains in $\beta$ PIX have been shown to function as independent units in vitro [27]. $\beta$ PIX also contains several PxxP motifs and a GIT1-binding domain, which is involved in the interaction with various cytoskeleton proteins, such as the G-proteincoupled receptor kinase-interacting target protein (GIT), the paxillin-kinase linker protein (p95PKL) and the cool-associated, tyrosine-phosphorylated protein [28-30]. A leucine zipper motif has been characterised at the $\mathrm{C}$-terminal end of the protein and shown to mediate the formation of $\beta$ PIX homodimers [31, 32].

The formation of $\beta$ PIX homodimers is important for its localisation to the cell periphery in order to drive the formation of membrane ruffles $[31,32]$. Thus, $\beta$ PIX is implicated in cytoskeleton remodelling important in cell migration [33]. Extracellular signals, e.g., from the PDGF receptor to cytoskeletal rearrangement are transmitted via $\beta$ PIX through the activation of Rac1 [24]. However, little is known about the associated signalling cascade and upstream activators of $\beta$ PIX leading to the activation of Rac1. Binding of $\beta$ PIX to PAK leads to Rac1 activation phenotype [34]. $\beta$ PIX binds also to p95PKL and GIT1, which link PAK to the central focal complex components, paxillin and focal adhesion kinase. These protein complexes promote focal adhesion turnover and Rac1-dependent mobility [28, 29].

In this study, we demonstrate that Abi- 1 and $\beta$ PIX interact in vitro and in vivo. Since Abi-1 and PIX play important roles in the PDGF-induced Rac1-dependent membrane ruffling, we investigated the biological function of the Abi-1 and $\beta$ PIX interaction in mouse fibroblasts in response to PDGF stimulation. Our results show that the interaction between Abi-1 and $\beta$ PIX is involved in PDGF-induced membrane dorsal ruffling.

\section{Materials and Methods}

\section{Constructs}

The different Abi-1 and $\beta$ PIX constructs are presented in Figure 1. The cDNA fragments encoding for $\beta$ PIX and Abi- 1 were cloned into EcoRI and XhoI sites of the pB42AD and pLexA yeast expression vectors (Clontech). The cDNA fragment encoding for Abi-1 fulllength (Abi-1 FL) sequence was cloned into the EcoRI and XhoI sites of the bacterial expression vector pGex-4T3 (Invitrogen), whereas the cDNA fragments encoding for the different $\beta$ PIX constructs were cloned into the BamHI and HindIII sites of the bacterial expression vector pET28c (Novagen). The different Abi-1 and $\beta$ PIX cDNA fragments were cloned into the NheI and XhoI sites of the mammalian expression vector pCEP4 (Invitrogen).

\section{Two-hybrid interaction}

The yeast two-hybrid assay was performed using the competent EGY48[p8op-LacZ] yeast strain prepared as follows. An overnight culture was used to inoculate a fresh culture at an $\mathrm{OD}_{600}$ of 0.1 , which was grown to an $\mathrm{OD}_{600}$ of 0.6 and harvested by centrifugation. Cells were washed in 1/10 culture volume Buffer A (1 M sorbitol, $1 \mathrm{mM}$ bicine, and $3 \%$ ethyleneglycol, $\mathrm{pH} 8.35)$, re-suspended in $1 / 100$ volume Buffer $\mathrm{A}$, aliquoted and stored first for $30 \mathrm{~min}$ at -20 ${ }^{\circ} \mathrm{C}$, and then frozen at $-70{ }^{\circ} \mathrm{C}$. Frozen competent yeast cells were transformed by heat shock with $1 \mu \mathrm{g}$ of the bait (pLexA) and prey (pB42AD) plasmid cDNAs, $5 \mu 1$ denaturised salmon's sperm (10 $\mu \mathrm{g} / \mu \mathrm{l})$, and $5 \mu \mathrm{l}$ histamine. After $5 \mathrm{~min}$ shaking at $37^{\circ} \mathrm{C}$ and addition of $1 \mathrm{ml}$ Buffer B (40\% PEG-1000 and $200 \mathrm{mM}$ bicine, $\mathrm{pH} 8.35)$, the sample was incubated for $1 \mathrm{~h}$ at $30^{\circ} \mathrm{C}$ with casual shaking. Cells were harvested by centrifugation, washed in $1 \mathrm{ml}$ Buffer C $(150 \mathrm{mM}$ $\mathrm{NaCl}, 10 \mathrm{mM}$ bicine, $\mathrm{pH}$ 8.35), re-suspended in $100 \mu 1 \mathrm{Buffer} \mathrm{C}$, and plated on selective medium: Trp-, His-, Ura-, galactose-raffinose$\mathrm{X}$-Gal-containing medium for the activation of the LacZ reporter gene and Trp-, His-, Ura-, glucose-X-Gal-containing medium for negative control. A positive interaction between the bait and prey proteins results in LacZ activation, conversion of X-Gal substrate, and selectable blue colonies.

\section{Cell cultures, transfections, and microinjection}

Transient expression of proteins for biochemical analysis and immunofluorescence was achieved by $\mathrm{Ca}^{2+}$ phosphate transfection. One day prior to transfection, $1 \times 10^{6} 293 \mathrm{~T}$ cells were seeded on $6 \mathrm{~cm}$ plates in DMEM (Life Technologies) with 10\% FCS, 1\% penicillin/streptomycin, $10 \mathrm{mM}$ HEPES (pH 7.4), and $4 \mathrm{mM}$ L-glutamine, or 40000 NIH3T3 cells were seeded on glass coverslips in 12-well plates in DMEM with $10 \%$ FCS, $1 \%$ penicillin/streptomycin, $1 \mathrm{mM}$ 
sodium pyruvate, and $4 \mathrm{mM}$ L-glutamine. Plasmid cDNAs were mixed with one volume of $250 \mathrm{mM} \mathrm{CaCl}_{2}$ and then with one volume of $2 \times \mathrm{HBS}$ (50 mM HEPES-NaOH (pH 7.0), $1.5 \mathrm{mM} \mathrm{Na}_{2} \mathrm{HPO}_{4}$, and $273 \mathrm{mM} \mathrm{NaCl}$ ). The preparation was added to the cells in medium supplemented with $25 \mu \mathrm{M}$ chloroquine (Sigma). One day posttransfection, NIH3T3 cells were grown in serum starvation medium ( $0.5 \%$ FCS) for $24 \mathrm{~h}$ and then stimulated with $15 \mathrm{ng} / \mathrm{ml}$ PDGF-BB (Upstate Biotechnology) for $10 \mathrm{~min}$ at $37{ }^{\circ} \mathrm{C}$ with $5 \% \mathrm{CO}_{2}$. Cells were fixed as described below.

Fifty ng/ $\mu$ l expression plasmid were microinjected into the nucleus of NIH3T3 cells seeded on glass coverslips as described above, using an Eppendorf microinjector. After $3 \mathrm{~h}$, cells were serum starved for $24 \mathrm{~h}$, treated with PDGF, and fixed as described below.

\section{Immunofluorescence}

Cells grown on glass coverslips were fixed with 3\% PFA for 30 min at $37{ }^{\circ} \mathrm{C}$ or overnight at $4{ }^{\circ} \mathrm{C}$. Cells were washed thrice with PBS, blocked and permeabilised with $0.2 \%$ Triton X-100, and $10 \%$ goat serum in PBS for $30 \mathrm{~min}$ at room temperature. Cells were incubated with primary antibodies: anti-HA Y-11 rabbit polyclonal (1:100, Santa Cruz), and/or anti-Flag M2 mouse monoclonal (1:2 500, Sigma), diluted in $3 \%$ goat serum and $0.05 \%$ Tween 20 in PBS for $1 \mathrm{~h}$ at room temperature. Cells were washed twice with PBS, blocked for $5 \mathrm{~min}$, and incubated with the secondary antibodies: $\mathrm{Cy} 3$-conjugated goat anti-mouse (1:100, Dianova), and/or Amca-conjugated anti-rabbit (1:100, Jackson), diluted as previously described, for $50 \mathrm{~min}$. Cells were washed twice with PBS, blocked for $5 \mathrm{~min}$, and incubated with Alexa Fluor 488 phalloidin for $45 \min$ (1:100, Molecular Probe). Preparations were analysed under a fluorescence microscope equipped with a $63 \times$ and a $100 \times$ oil immersion objectives (Leica), and a digital camera. Images were processed using the TCS Software and Adobe Photoshop.

\section{Immunoprecipitation and immunoblotting}

For precipitation of endogenous Abi-1, NIH3T3 cells were grown in serum starvation medium $(0.5 \% \mathrm{FCS})$ for $24 \mathrm{~h}$ and were then stimulated with $15 \mathrm{ng} / \mathrm{ml}$ PDGF-BB for $10 \mathrm{~min}$ at $37^{\circ} \mathrm{C}$ with $5 \% \mathrm{CO}_{2}$. Cells were lysed in $100 \mathrm{mM} \mathrm{NaCl}, 10 \mathrm{mM}$ sodium phosphate buffer, $2 \mathrm{mM}$ EGTA, $1 \mathrm{mM}$ EDTA, $10 \mathrm{mM} \mathrm{NaF}, 10 \mathrm{mM} \mathrm{Na} 2 \mathrm{P}_{2} \mathrm{O}_{7}, 0.5 \%$ deoxycholate, $0.05 \%$ SDS, $5 \%$ glycerol, $1 \%$ NP-40, plus protease inhibitor mix ( $1 \mu \mathrm{g} / \mathrm{ml}$ aprotinin, $0.5 \mu \mathrm{g} / \mathrm{ml}$ leupeptin, $1 \mathrm{mM}$ pefabloc, and $10 \mu \mathrm{M}$ pepstatin), $\mathrm{pH}$ 7.2. Lysates were incubated or not with Abi-1 monoclonal antibody (generous gift from Giorgio Scita, Istituto FIRC di Oncologia Molecolare, Milan, Italy). In case of immunoprecipitation of overexpressed proteins, cells were harvested $48 \mathrm{~h}$ post-transfection, washed with cold PBS, and re-suspended in $600 \mu \mathrm{l}$ Single-Detergent-Buffer-1 (20 mM Tris-HCl, pH 7.5, $150 \mathrm{mM} \mathrm{NaCl}$, and $1 \%$ NP-40 with protease inhibitor mix) on ice for $10 \mathrm{~min}$. Cells were then passed through a syringe twice and centrifuged at 13000 $\mathrm{rpm}$. for $15 \mathrm{~min}$ at $4{ }^{\circ} \mathrm{C}$. Cell lysates were pre-incubated and rotated with the indicated agarose-coupled antibody, anti-HA (Santa Cruz) or anti-Flag (M2, Sigma), overnight at $4{ }^{\circ} \mathrm{C}$. Immunoprecipitated proteins bound to the agarose matrix were then washed in SingleDetergent-Buffer- 1 without protease inhibitors, boiled in $2 \times$ sample solution, separated in SDS-PAGE, and detected by immunoblotting with the antibodies specific for the protein's tag. Anti-HA Y-11 rabbit polyclonal (1:1 000), anti-Flag M2 mouse monoclonal (1:5 000), goat anti-rabbit peroxidase, and goat anti-mouse peroxidase (1:3000, Amersham Life Science) were used.

\section{In vitro translation}

In vitro translation was performed using the $\mathrm{TnT}^{\circledR}$ Coupled Reticulocyte Lysate System (Promega) according to the manufacturer's protocol. For a reaction in $50 \mu \mathrm{H}_{2} \mathrm{O}, 1 \mu \mathrm{g}$ plasmid cDNA coding for different deletion constructs of PIX and $5 \mu 1\left[{ }^{35} \mathrm{~S}\right]$ methionine $(10 \mu \mathrm{Ci} / \mu \mathrm{l}$, Amersham/Pharmacia) were mixed. Protein translation was reported by SDS-PAGE, Coomassie staining, and exposure of the gels to films.

\section{GST protein expression, purification, and GST pull-down} assay

GST-Abi-1 full-length (GST-Abi-1 FL) and GST were expressed in BL21-CodonPlus (DE3)-RP Escherichia coli strain (Stratagene) by the addition of $0.5 \mathrm{mM}$ IPTG. For cell lysis, bacteria were resuspended on ice in $2 \mathrm{ml}$ cold PBS with protease inhibitor mix and sonicated four times for $30 \mathrm{~s}$. Purification of GST-Abi-1 FL and GST was performed with a $50 \%$ gluthatione sepharose $4 \mathrm{~B}$ matrix (Pharmacia) at $4{ }^{\circ} \mathrm{C}$ and rotated for $2 \mathrm{~h}$. The matrix containing the bound protein was washed twice with PBS. The proteins were eluted with $20 \mathrm{mM}$ gluthatione, $50 \mathrm{mM}$ Tris- $\mathrm{HCl}$ (pH 8.0), $100 \mathrm{mM} \mathrm{NaCl}$, and dialysed in PBS overnight at $4^{\circ} \mathrm{C}$. The dialysed protein was shock frozen and stored at $-70^{\circ} \mathrm{C}$. For pull-down assay the purified GSTAbi-1 FL and GST were bound to gluthatione sepharose 4B matrix, as described previously, washed with cold PBS, and incubated with the different in vitro translated $\beta$ PIX proteins for $30 \mathrm{~min}$ at $4{ }^{\circ} \mathrm{C}$ with shaking. Samples were centrifuged and pellets washed thrice with the Single-Detergent-Buffer-2 (20 mM Tris-HCl, pH 7.5, 500 $\mathrm{mM} \mathrm{NaCl}$, and $1 \% \mathrm{NP}-40$ with protease inhibitor mix). Pellets were re-suspended in $50 \mu 1$ Single-Detergent-Buffer-2, $10 \mu 12 \times$ sample solution and samples were analysed by SDS-PAGE. Then, the gels were stained with Coomassie, dried, and exposed overnight.

\section{Results}

\section{A new interaction between $\beta P I X$ and $A b i-1$}

In order to identify new interaction partners for PAKs, a yeast two-hybrid screen was performed, which resulted in the isolation of Abi-1 and $\beta$ PIX (unpublished data). We also performed yeast two-hybrid screens to find out whether Abi- 1 and $\beta$ PIX not only interact with PAKs but may also be with each other. Different fragments of $\beta$ PIX were fused to the activation domain of the $\mathrm{pB} 42 \mathrm{AD}$ vector. The Abi-1 FL sequence was fused downstream to the DNA-binding domain of the pLexA vector (Figure 1). The expression of all constructs was verified by Western blot analysis (not shown). As a negative control, the different $\beta$ PIX fusion proteins and the Abi-1 FL fusion protein were each tested for potential self-activation of the LacZ reporter gene and for potential interactions with a non-related protein, lamin C or caspase- 9 , respectively. No self-activation by any of these fusion proteins was detected, nor did these fusions show any interaction with non-related negative control proteins (data not shown). To prove that the system is useful for detecting proteins interacting with $\beta$ PIX, the interaction of $\beta$ PIX with its known partner PAK2 [25] was tested and verified. 


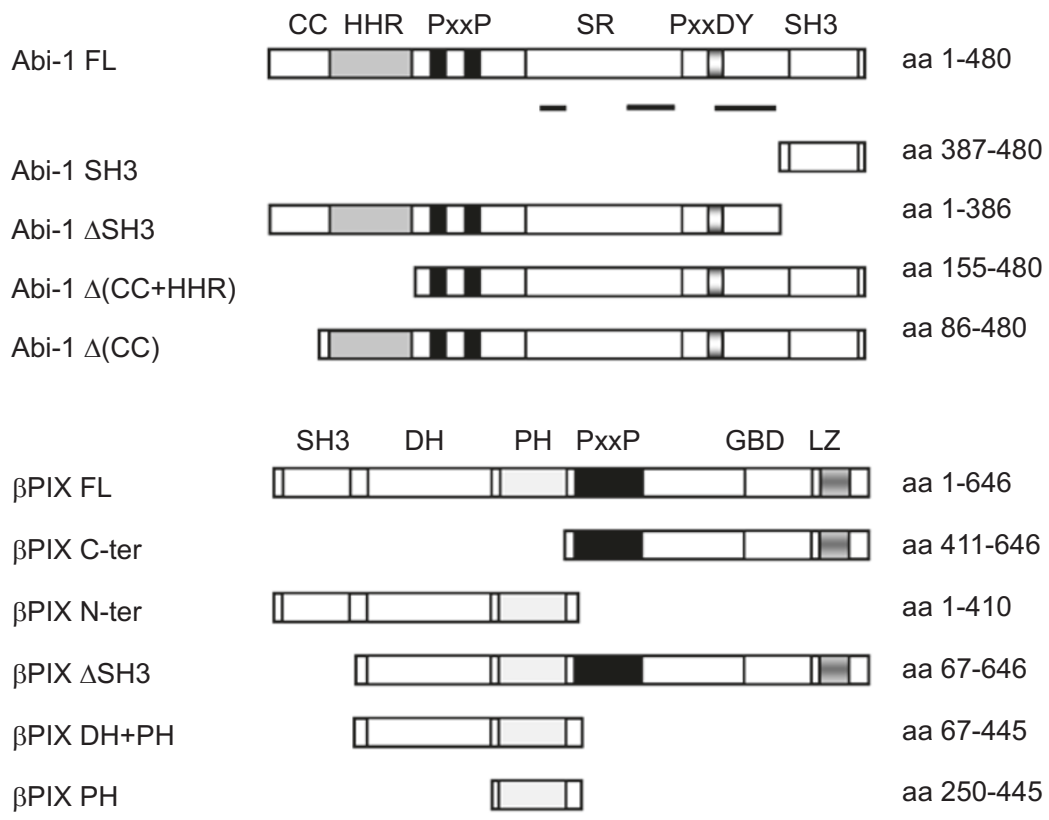

Figure 1 Abi-1 and $\beta$ PIX constructs. Schematic representation of the various constructs used in this work. For yeast two-hybrid analysis, the indicated DNA fragments were cloned into the $\mathrm{pB} 42 \mathrm{AD}$ and the pLexA vectors, downstream of the activation domain and the DNA-binding domain, respectively. For pull-down assays, Abi-1 constructs were cloned into the pGex-4T3 bacterial expression vector containing an N-terminal GST tag, and $\beta$ PIX coding sequences were cloned into the pET28c vector. These constructs were also cloned into the pCEP4 mammalian expression vector for immunoprecipitation experiments and subcellular localisation studies of Abi-1 and $\beta$ PIX. $\beta$ PIX constructs were cloned with an N-terminal HA tag and Abi- 1 constructs with a N-terminal Flag tag. aa: amino acids; $\Delta$ : deletion; FL: full-length; CC: coiled-coiled domain; HHR: homeo-domain homologous region; PxxP: proline-rich region; PxxDY: unconventional proline-rich region; SR: serine-rich region; SH3: Src Homology 3 domain; C-ter: C-terminus; N-ter: N-terminus; DH: Dbl homology domain; PH: pleckstrin homology domain; GBD: GIT1-binding domain; and LZ: leucine zipper. Black underlined lines in Abi-1 represented sequences rich in proline, glutamic acid, serine, and threonine (PEST sequences).

The different $\beta$ PIX constructs were co-transformed with the Abi-1 FL construct into yeast and assayed for interaction. Full-length Abi-1 and full-length $\beta$ PIX clearly interacted as indicated by the positive $\beta$-galactosidase reaction (Figure 2A). Neither the $\beta$ PIX hybrid protein containing the N-terminal domain from amino acids 1 to 410 nor the $\beta$ PIX hybrid proteins containing the $\mathrm{DH}$ and $\mathrm{PH}$ domains or the $\mathrm{PH}$ domain alone interacted with Abi-1. However, an interaction was detected with the $\beta$ PIX hybrid protein containing a deletion of the SH3 domain and with the $\beta$ PIX hybrid protein containing the $\mathrm{C}$-terminal domain from amino acids 410 to 646 . Thus, in this assay the interaction of $\beta$ PIX with Abi-1 was mediated by the C-terminus of $\beta P I X$.

Yeast two-hybrid assays were also performed with different constructs of Abi-1 described in Figure 1. These constructs were co-transformed in yeast with the $\beta$ PIX full-length construct, in order to define the domain of interaction in Abi-1. Surprisingly, only full-length Abi-1 interacted with $\beta$ PIX (Figure $2 \mathrm{~B}$ ), suggesting that the in- teraction between Abi-1 and $\beta$ PIX may require a particular conformation of Abi-1, which is destroyed in Abi-1 deletion proteins.

\section{Interaction between $\beta P I X$ and Abi-1 in vitro}

The interaction between Abi- 1 and $\beta$ PIX observed in the yeast two-hybrid assay was confirmed using in vitro GST pull-down assays. GST-Abi-1 FL was constructed and used to pull down various radioactively labelled $\beta$ PIX constructs. As shown in Figure 3, the interaction between Abi-1 and $\beta$ PIX full-length was confirmed. Neither the $\mathrm{N}$-terminal fragment, nor the fragment containing the $\mathrm{PH}$ domain nor the fragment containing the $\mathrm{DH}$ and $\mathrm{PH}$ domains of $\beta$ PIX interacted with full-length Abi-1. However, Abi-1 FL interacted with the C-terminal fragment and the construct that lacked the SH3 domain of $\beta$ PIX. These results are consistent with the results obtained in the yeast two-hybrid analyses indicating that the C-terminus of $\beta$ PIX mediates the interaction between $\beta$ PIX and Abi-1 in vitro. 
A
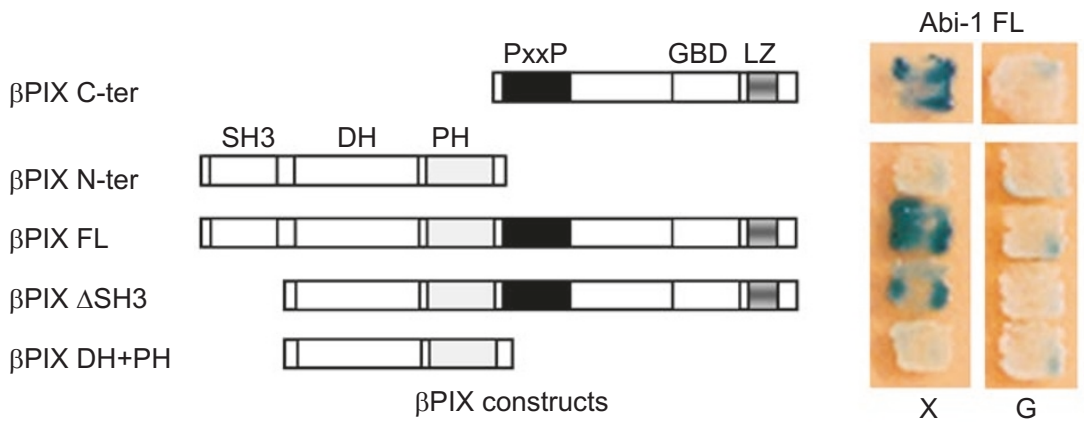

B

Abi-1 FL

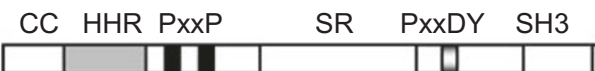

Abi-1 SH3

Abi-1 $\Delta \mathrm{SH} 3$

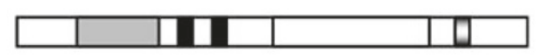

Abi-1 $\Delta(\mathrm{CC}+\mathrm{HHR})$

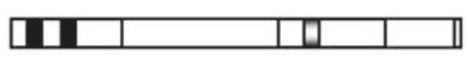

Abi-1 constructs

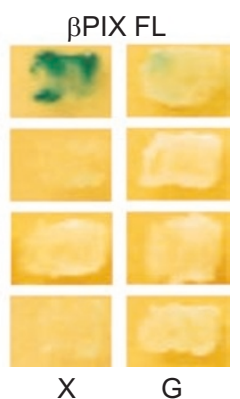

Figure 2 Identification of an interaction between Abi-1 and $\beta$ PIX. The pB42AD vector expressing the indicated $\beta$ PIX fusions and the pLexA vector expressing the indicated Abi-1 fusions were co-transformed into the yeast strain EGY48[p8op-LacZ] to test for interaction in a two-hybrid assay as described. (A) Interaction of $\beta$ PIX or its fragments with full-length Abi-1 on X-Gal- (X) or glucose- $(G)$ containing medium. (B) Interaction of Abi-1 or its fragments with full-length $\beta$ PIX on X-Gal- $(X)$ or glucose- $(G)$ containing medium. The blue coloured colonies were observed when interaction had occurred. Clones containing the bait and the prey plasmids were amplified and the expression of the activation domain fusion proteins was repressed by the presence of glucose (G) in the medium. Abbreviations are explained in the legend of Figure 1.

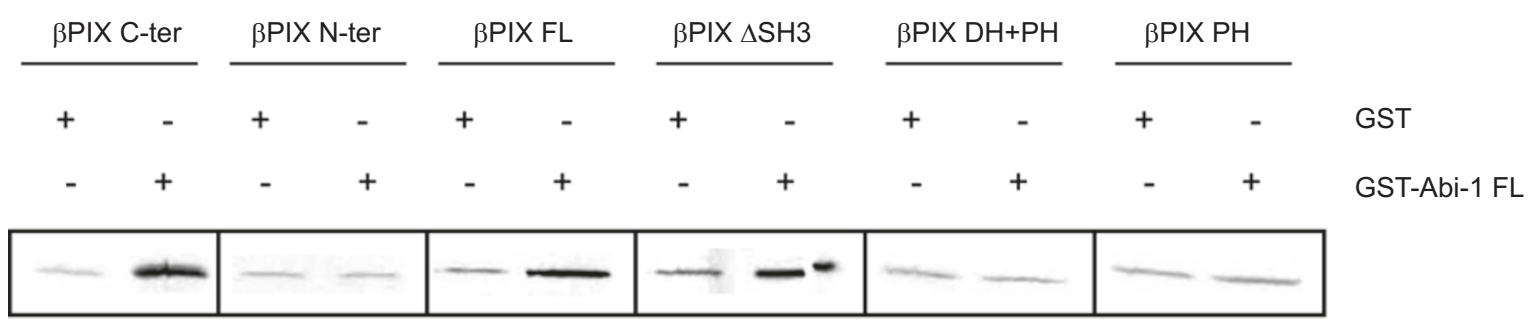

Figure 3 Characterisation of the interaction between Abi-1 and $\beta$ PIX in vitro. The interaction between full-length Abi-1 and fulllength $\beta$ PIX or its fragments was assayed in pull-down experiments using a GST-Abi-1 full-length (GST-Abi-1 FL) fusion protein. The full-length Abi-1 protein was fused to the C-terminus of the GST tag and expressed from the pGex-4T3 bacterial expression vector. GST alone or GST-Abi-1 FL were coupled to sepharose and then incubated with radioactively labelled in vitro translated full-length $\beta$ PIX or its fragments (for the description of constructs see Figure 1). Samples were eluted and separated by SDS-PAGE; gels were dried and exposed to an X-ray film overnight.

\section{Interaction between $\beta$ PIX and Abi-1 in vivo}

In order to determine whether Abi-1 and $\beta$ PIX could interact in vivo, co-immunoprecipitation of endogenous Abi-1 and $\beta$ PIX was performed in NIH3T3 cells. NIH3T3 cells were serum starved for $24 \mathrm{~h}$ and treated with PDGF before Abi-1 was precipitated with a monoclonal Abi-1 antibody. $\beta$ PIX clearly co-precipitated with Abi-1 in PDGFtreated cells (Figure 4A), suggesting an interaction of the 
two proteins in these cells. Since the $\beta$ PIX anti-serum was inefficient in precipitating mouse $\beta$ PIX from NIH3T3 cells, the human $\beta$ PIX was overexpressed and immunoprecipitated from HeLa cells. Endogenous Abi-1 co-precipitated with $\beta$ PIX (Supplement 1), suggesting that both proteins are organised in the same complex.

To map the domain of interaction in Abi- 1 and $\beta$ PIX, 293 T cells were double transfected either with Flag-Abi-1 FL and different HA-tagged $\beta$ PIX constructs (Figure 4B) or with $\beta$ PIX full-length and different Abi-1 constructs (Figure $4 C)$. The full-length HA- $\beta$ PIX protein co-immunoprecipitated with the full-length Flag-Abi-1 protein. Interestingly, co-transfections of different $\beta$ PIX constructs with Abi-1 FL showed that the $\beta$ PIX N-terminal fragment, the construct lacking the SH3 domain, and the construct containing the DH and PH domains of $\beta$ PIX interacted with Abi-1 FL.
The C-terminal construct of $\beta$ PIX, which interacted in vitro with Abi-1 FL (Figure 3), did not interact with Abi-1 FL in vivo (Figure 4B). However, in confirmation of the yeast two-hybrid results, none of the Abi-1 deletion constructs interacted with $\beta$ PIX full-length (Figure $4 \mathrm{C}$ ). Thus, whereas further evidence could be obtained that a conformational epitope of Abi-1 is involved in the interaction with $\beta$ PIX, the $\beta$ PIX fragments interacting with Abi- 1 in vitro and in vivo differ.

\section{Abi-1 and $\beta P I X$ co-localise to membrane dorsal ruffles in} PDGF-induced NIH3T3 cells

Rac1 regulates PDGF-induced membrane dorsal ruffling [5]. It has been demonstrated that Abi-1 protein induces membrane dorsal ruffling in fibroblasts through Rac1 activation in response to PDGF [19]. Overexpression of the

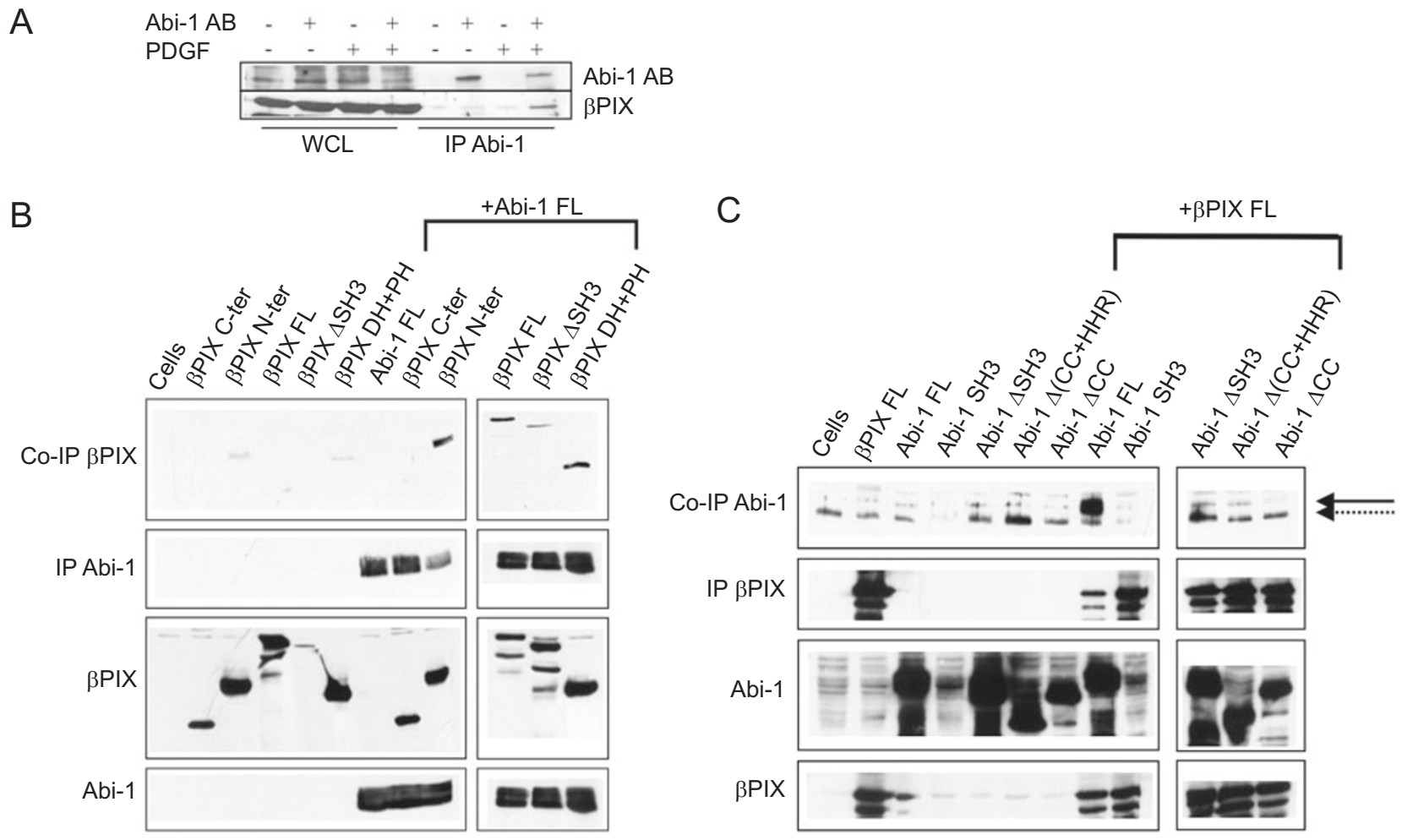

Figure 4 In vivo interaction between Abi-1 and $\beta$ PIX. (A) NIH3T3 were serum starved and treated with (+) or without (-) PDGF before lysis and immunoprecipitation of Abi- 1 with monoclonal antibody. The levels of precipitated Abi- 1 and co-precipitated $\beta$ PIX were monitored in the lysates (WCL) and the Abi-1 immunoprecipitations (IP Abi-1) with (+) and without (-) Abi-1 antibody (Abi-1 $\mathrm{AB})$. (B, C) 293T cells were mock transfected (cells) or transfected with $5 \mu \mathrm{g}$ of each of the different deletion constructs of $\beta$ PIX and Abi-1 FL in (B) or with each of the different deletion constructs of Abi-1 and BPIX FL in (C). (B) Flag-Abi-1 FL was immunoprecipitated (IP) with the anti-Flag antibody from the total cell lysates, and protein complexes were detected by immunoblotting with anti-Flag and anti-HA antibodies for Flag-Abi-1 FL and HA- $\beta$ PIX, respectively. (C) HA- $\beta$ PIX FL was immunoprecipitated with the anti-HA antibody from the total cell lysates and protein complexes were detected by immunoblotting as described above. The black arrow points to Flag-Abi-1 FL and the broken arrow to the antibody heavy chain. The two bottom panels in (B) and (C) show the expression of the diverse constructs, the top panels show the immunoprecipitated or co-immunoprecipitated proteins. The diverse constructs for Abi-1 and $\beta$ PIX are represented in Figure 1. 
$\beta$ PIX protein, known to function as a GEF protein for Rac1, induces membrane dorsal ruffling in fibroblasts through Rac1 [24, 31, 34]. In order to investigate the subcellular distribution of Abi- 1 and $\beta$ PIX in NIH3T3 cells upon PDGF treatment, the expression vectors encoding, either for $\beta$ PIX full-length or Abi-1 FL, were microinjected into fibroblasts. Serum-starved cells were induced with PDGF, and $\beta$ PIX and Abi-1 were visualised by indirect immunofluorescence. Abi-1 localised in the cytoplasm in non-treated cells (Figure 5B) and to membrane dorsal ruffles in PDGF-treated cells (Figure 5D). The same localisation pattern was observed for $\beta$ PIX (Figure 5E-5H), seen localised in the cytoplasm (Figure 5F), and then at membrane dorsal ruffles in response to PDGF treatment (Figure 5H).

Then, the subcellular distribution of Abi- 1 and $\beta$ PIX full-length proteins co-expressed in NIH3T3 cells was analysed. Abi- 1 and $\beta$ PIX exhibited a similar diffuse staining pattern in the cytoplasm of non-treated cells (Figure 5J, $5 \mathrm{~K}$, and $5 \mathrm{~L}$ ), suggesting that both proteins localise in the same compartment. In PDGF-treated cells, a substantial portion of Abi- 1 and $\beta$ PIX localised to membrane dorsal ruffles (Figure $5 \mathrm{~N}, 5 \mathrm{O}$, and $5 \mathrm{P}$ ), compared to the diffuse cytoplasmic localisation of Abi-1 and $\beta$ PIX in serumstarved, untreated cells (Figure 5J, 5K, and 5L). Taken together, the co-localisation of Abi- 1 and $\beta$ PIX to sites of membrane dorsal ruffling and the interaction between Abi-1
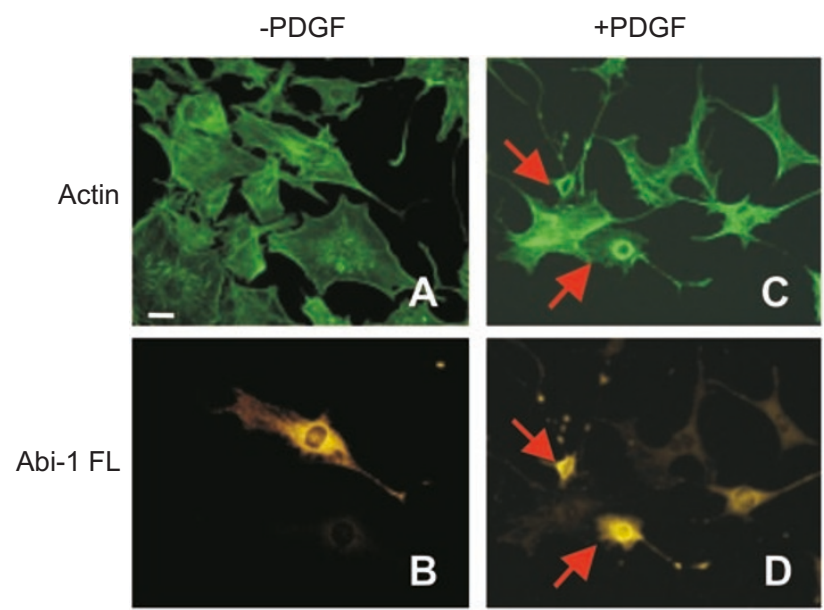

B
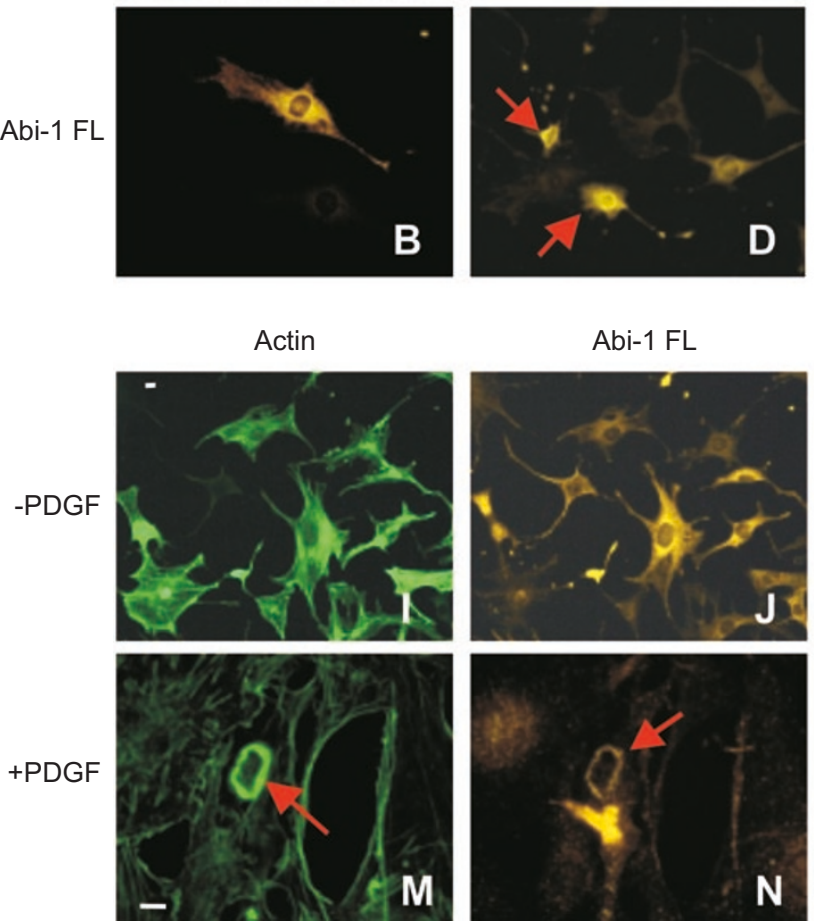

-PDGF
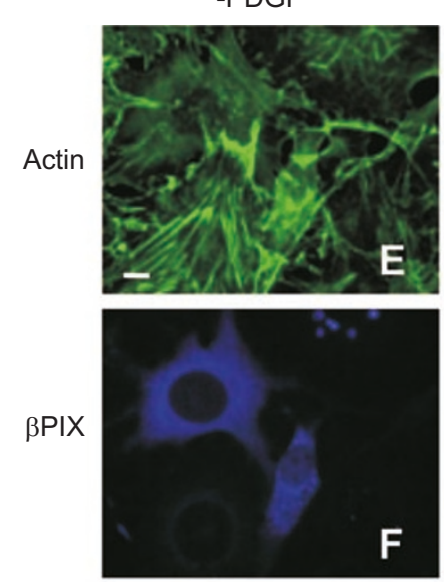

$\beta P I X F L$

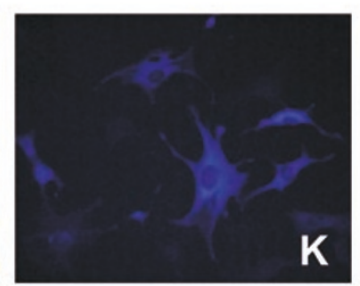

Overlay Abi-1 FL- $\beta$ PIX FL

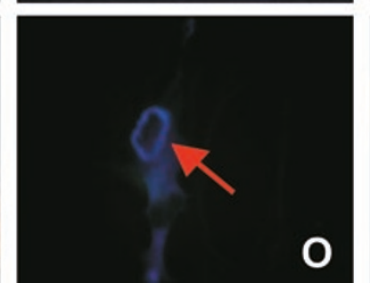

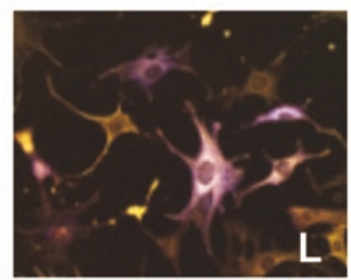

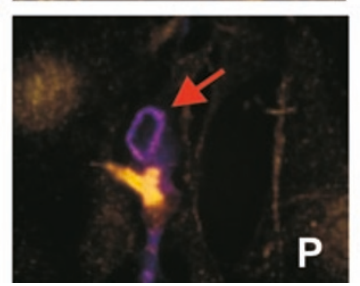

Figure 5 Subcellular localisation of Abi-1 and $\beta$ PIX upon PDGF induction. Abi-1 (A-D) and $\beta$ PIX (E-H) localise to membrane dorsal ruffles. Nuclei of mouse fibroblasts (NIH3T3) were microinjected with $50 \mathrm{ng} / \mu 1$ of the expression vector for Abi-1 full-length (Abi-1 FL) or $\beta$ PIX full-length ( $\beta$ PIX FL). Cells were serum starved and treated with $(\mathbf{C}, \mathbf{D}, \mathbf{G}, \mathbf{H})$ or without $(\mathbf{A}, \mathbf{B}, \mathbf{E}, \mathbf{F})$ PDGF. Abi-1 and $\beta$ PIX co-localise to membrane dorsal ruffles (I-P). Nuclei of NIH3T3 were co-microinjected with expression vectors for $\beta$ PIX FL and Abi-1 FL. Cells were serum-starved and treated with (M, N, O, P) or without (I, J, K, L) PDGF. Cells were fixed and stained for immunofluorescence with Alexa-conjugated phalloidin to detect F-actin (green), with the anti-Flag antibody to detect Abi-1 (yellow), and with the anti-HA antibody to detect $\beta$ PIX (blue). Arrows point to membrane dorsal ruffles. Bars, $10 \mu \mathrm{m}$. 
and $\beta$ PIX suggest a role for this interaction in the formation of membrane dorsal ruffles in response to PDGF.

The interaction between Abi-1 and $\beta P I X$ is involved in the formation of PDGF-induced membrane dorsal ruffles

To examine the involvement of the interaction between Abi- 1 and $\beta$ PIX in the induction of membrane dorsal ruffles in PDGF-treated NIH3T3 cells, the effect of co-expression of Abi-1 FL, either with the N-terminal or the C-terminal fragments of $\beta$ PIX, was investigated.

The effects of the N-terminal and C-terminal fragments of $\beta$ PIX were first studied. Therefore, the vector encoding for the $\mathrm{N}$-terminal or the $\mathrm{C}$-terminal fragment of $\beta$ PIX was transfected in NIH3T3 cells. Serum-starved transfected cells were treated with PDGF and overexpressed proteins were visualised by immunofluorescence microscopy. As shown in Figure 6A-6H, the N-terminal fragment of $\beta$ PIX (Figure 6B) as well as the $\mathrm{C}$-terminal fragment (Figure 6F) localised in the cytoplasm of serum-starved, non-treated cells. Cells overexpressing either the $\mathrm{N}$-terminal fragment (Figure 6D) or the $\mathrm{C}$-terminal fragment of $\beta$ PIX (Figure $6 \mathrm{H})$ did not induce membrane dorsal ruffling after PDGF treatment. Thus, upon treatment with PDGF, the N-terminal and the $\mathrm{C}$-terminal fragment of $\beta$ PIX inhibited the formation of membrane dorsal ruffles, suggesting both domains are required for this PDGF-mediated response.

Then, NIH3T3 cells were co-transfected either with the vectors encoding for Abi-1 FL and the N-terminal fragment of $\beta$ PIX (Figure 6I-6P) or with the vectors encoding for Abi-1 FL and the C-terminal fragment of $\beta$ PIX (Figure 6Q-6X). The number of cells forming ruffles upon PDGF stimulation was similar in cells expressing either Abi-1 FL or $\beta$ PIX full-length alone ( $80 \%$ and $83 \%$, respectively). Cells co-expressing Abi-1 FL and the N-terminal fragment of $\beta$ PIX did not exhibit morphological changes in response to PDGF (Figure 6M, 6N, 6O, and 6P). Whereas $78.4 \%$ of cells overexpressing Abi-1 FL and $\beta$ PIX full-length formed membrane dorsal ruffles after PDGF induction, only $11.74 \%$ of cells overexpressing Abi-1 FL and the $\beta$ PIX N-terminal fragment showed this phenotype (Figure $6 Y)$. A similar effect was observed with cells co-expressing Abi- $1 \mathrm{FL}$ and the C-terminal fragment of $\beta$ PIX (Figure $6 \mathrm{U}, 6 \mathrm{~V}, 6 \mathrm{~W}$, and $6 \mathrm{X})$, since only $5.5 \%$ of the cells showed this phenotype (Figure 6Y). Thus, both the N-terminal and $\mathrm{C}$-terminal fragment of $\beta$ PIX functioned in a dominantnegative manner and blocked PDGF-induced membrane dorsal ruffling.

\section{Discussion}

We report here a novel interaction between Abi-1 and $\beta P I X$. This interaction was shown in yeast two-hybrid experiments, in vitro, by GST pull-down experiments and, in vivo, by co-immunoprecipitation of the endogenous and overexpressed proteins.

Our data indicate that the C-terminal fragment of $\beta$ PIX interacts with Abi- 1 in vitro. The $\mathrm{C}$-terminal fragment of $\beta$ PIX contains different domains including several PxxP motifs, whereas Abi- 1 contains in its C-terminal part an SH3 domain. The fact that $\mathrm{SH} 3$ domain and PxxP motifs are typical interaction modules in protein-protein interactions [35] suggests that $\beta$ PIX interacts with the $\mathrm{SH} 3$ domain of Abi-1 through the PxxP motifs present in the C-terminal region. This has been shown for another binding partner of Abi-1, the Sos-1 protein [19, 22].

Interestingly, Abi-1 and Sos-1 interact via different domains in vitro and in vivo. In vivo, Sos-1 interacts with the N-terminus of Abi-1 and not with the SH3 domain contained in the C-terminus [36]. We find a similar case for the interaction of $\beta$ PIX and Abi-1. In contrast to the results of initial in vitro binding studies, in vivo Abi-1 co-immunoprecipitated with the $\mathrm{N}$-terminal fragment of $\beta$ PIX and not with the C-terminal fragment. Nevertheless, it cannot be excluded that the $\mathrm{C}$-terminal fragment of $\beta \mathrm{PIX}$

Figure 6 Functional interaction of Abi-1 and $\beta$ PIX. (A-H) The N-terminal fragment ( $\beta$ PIX N-ter) and C-terminal fragment ( $\beta$ PIX C-ter) of $\beta$ PIX inhibit membrane dorsal ruffle formation in response to PDGF. NIH3T3 were transfected with the expression vector for $\beta$ PIX N-ter (A-D) or $\beta$ PIX C-ter (E-H) for 1 day. Cells were serum starved and treated with $(\mathbf{C}, \mathbf{D}, \mathbf{G}, \mathbf{H})$ or without (A, B, E, F) PDGF. (I-P) Co-expression of the N-terminal fragment of $\beta$ PIX with Abi-1 full-length (Abi-1 FL) inhibits membrane dorsal ruffle formation in response to PDGF. NIH3T3 were co-transfected with expression vectors for $\beta$ PIX N-ter and Abi-1 FL for 1 day. Cells were serum-starved and treated with $(\mathbf{M}, \mathbf{N}, \mathbf{O}, \mathbf{P})$ or without $(\mathbf{I}, \mathbf{J}, \mathbf{K}, \mathbf{L})$ PDGF. (Q-X) Co-expression of the C-terminal fragment of $\beta$ PIX with Abi-1 FL inhibits membrane dorsal ruffle formation in response to PDGF. NIH3T3 were co-transfected with expression vectors for $\beta$ PIX C-ter and Abi-1 FL for 1 day. Cells were serum starved and treated with $(\mathbf{U}, \mathbf{V}, \mathbf{W}, \mathbf{X})$ or without $(\mathbf{Q}, \mathbf{R}, \mathbf{S}, \mathbf{T})$ PDGF. Cells were fixed and stained with Alexa-conjugated phalloidin to detect F-actin (green), with the anti-Flag antibody to detect Abi-1 (yellow), and with the anti-HA antibody to detect $\beta$ PIX (blue). Arrows point to membrane dorsal ruffles and asterisks identify the transfected cell. Bars, $10 \mu \mathrm{m}$. (Y) Quantification of the experiments shown from (I) to (X). NIH3T3 cells expressing Abi-1 FL and the indicated full-length or truncated $\beta$ PIX proteins were stimulated with PDGF, and were scored for the presence or absence of membrane dorsal ruffling. Data represent the mean of three independent experiments. Bars indicate the standard errors. 


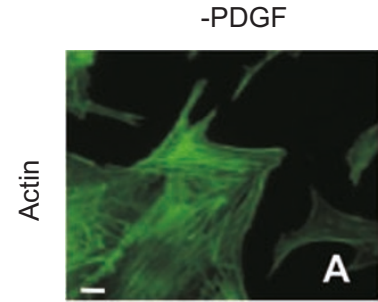

+PDGF

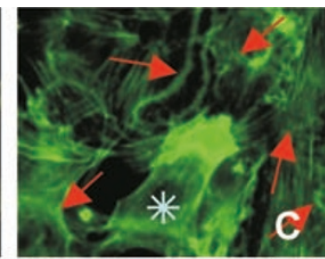

B

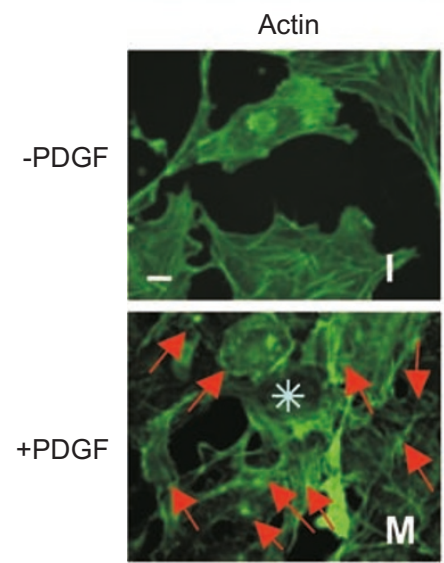

Actin
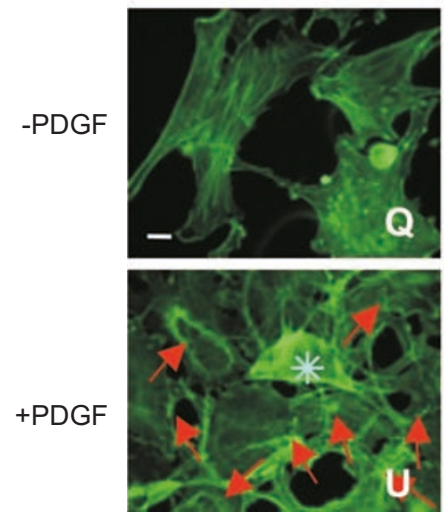

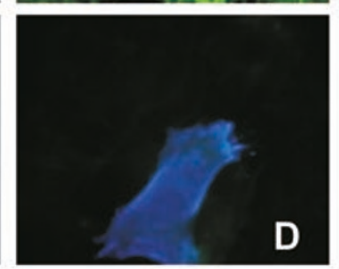

Abi-1 FL

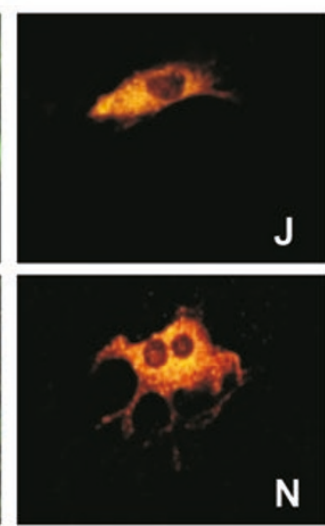

Abi-1 FL
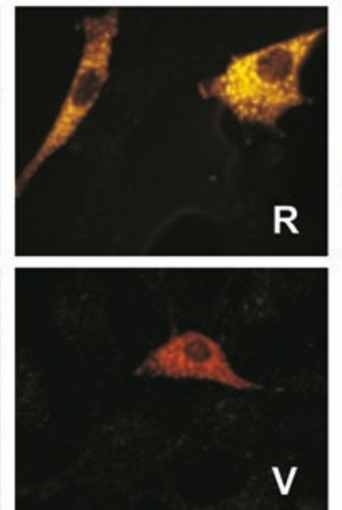

-PDGF
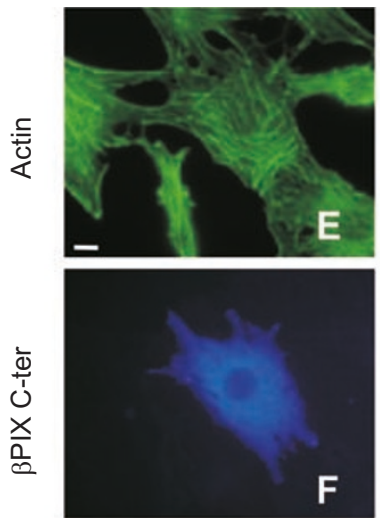

$\beta P I X N$-ter

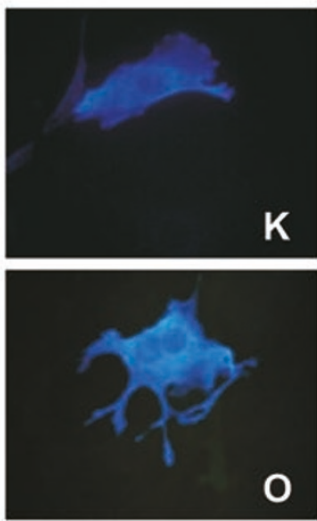

$\beta P I X$ C-ter

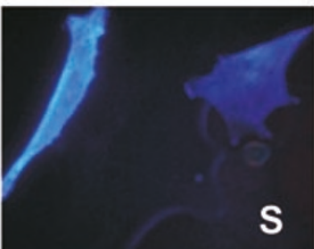

S

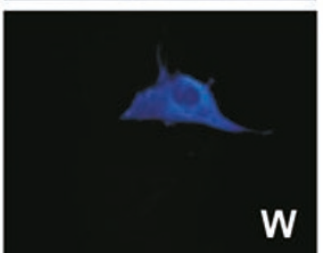

+PDGF
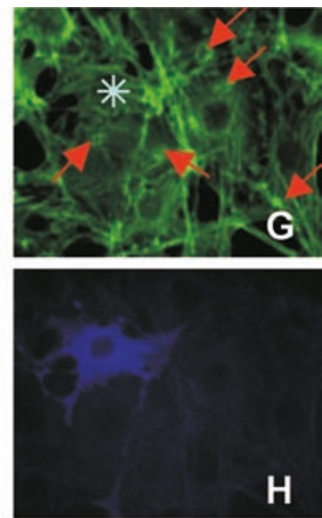

Overlay Abi-1 FL- $\beta$ PIX N-ter

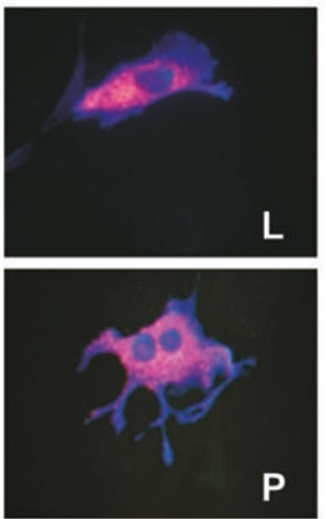

Overlay Abi-1 FL- $\beta$ PIX C-ter
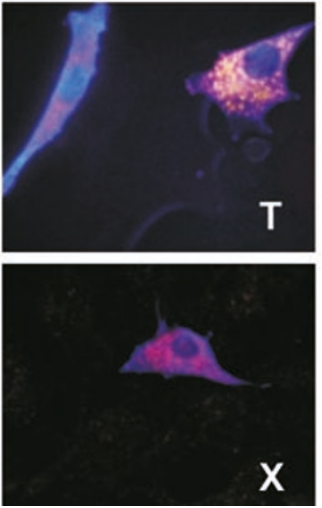

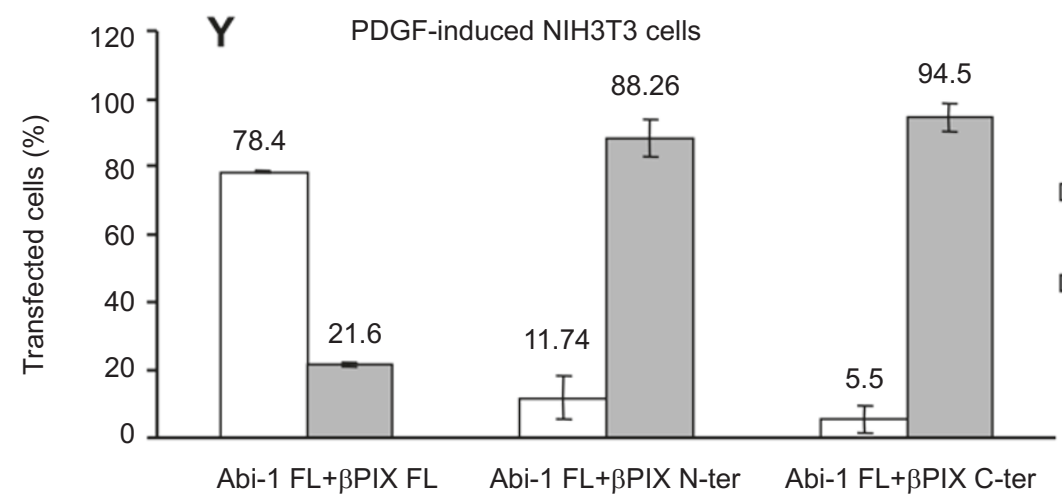

$\square$ Cells forming membrane dorsal ruffles

$\square$ Cells without phenotype 
interacts with Abi-1 in vivo. The expressed C-terminal fragment may acquire a conformation different from the full-length protein, which is not recognised by Abi-1, or another protein may have masked the Abi-1-interacting domain in the $\mathrm{C}$-terminus of $\beta$ PIX.

The results obtained in vivo showed that the $\mathrm{SH} 3$ domain of $\beta$ PIX is not required for an interaction with Abi-1, while the fragment of $\beta$ PIX containing the $\mathrm{DH}$ and $\mathrm{PH}$ domains bound to Abi-1. DH domains contain the GEF activity, whereas $\mathrm{PH}$ domains are known to bind specifically to phosphatidylinositol-4, 5-biphosphate, which targets the protein to membranes [37]. This is now realised to be a property of a small number of $\mathrm{PH}$ domain-containing proteins, which bind phosphoinositides only very weakly. This suggests that either most $\mathrm{PH}$ domains require assistance from other parts of the protein in order to achieve their function or the physiological ligands of $\mathrm{PH}$ domains are not limited to phosphoinositides [38].

In the present study, it was not possible to define the domain of interaction in Abi-1 in vitro and in vivo. As soon as the full-length protein Abi-1 is deleted either in its $\mathrm{N}$-terminus or C-terminus, no interaction was detected with $\beta$ PIX. It can be hypothesised that deletions in Abi-1 destroy a conformation of the protein necessary for binding to $\beta$ PIX. Another possible explanation for this observation is the limited stability of the truncated Abi-1 proteins. Several bands of low molecular weight reacted with the anti-Flag antibody (Figure 4C) indicating the presence of degraded Abi-1 products, an observation made before by others [13]. It is possible that the rapid intracellular proteolysis is mediated by the PEST sequences present in Abi-1, which can function as a signal for proteasomemediated degradation.

The co-localisation of Abi- 1 and $\beta$ PIX in the cytoplasm confirms the relevance of the interaction between the two proteins. After induction with PDGF, both Abi-1 and $\beta$ PIX co-localised to membrane dorsal ruffles. To study the function of the interaction between Abi- 1 and $\beta$ PIX in PDGF-induced membrane dorsal ruffles in NIH3T3 cells, the subcellular localisation and the effects of overexpressed $\beta$ PIX deletion constructs and Abi-1 FL were investigated. The N-terminal fragment of $\beta$ PIX overexpressed with Abi$1 \mathrm{FL}$ in PDGF-induced cells shows a diffuse cytoplasmic localisation and abrogates membrane dorsal ruffling. The same phenotype was observed when the C-terminal fragment of $\beta$ PIX was overexpressed with Abi-1 FL.

What is the possible significance of the effect of the $\mathrm{N}$-terminal fragment of $\beta$ PIX? The N-terminal construct containing the domain of interaction with Abi-1, in vivo, may act in a dominant-negative manner, abrogating the interaction between Abi- 1 and endogenous $\beta$ PIX. The diffuse cytoplasmic localisation of the $\mathrm{N}$-terminal fragment of $\beta P I X$ after PDGF induction could result from the sequestration of downstream effectors molecules essential for membrane dorsal ruffling. Additionally, $\beta$ PIX homodimerise in vitro and in vivo through its leucine zipper domain located in the C-terminal extremity of the protein [32]. The ability of $\beta$ PIX to homodimerise is necessary for the $\beta$ PIX-mediated membrane dorsal ruffle formation in NIH3T3 fibroblasts in response to PDGF [31]. Although the N-terminal fragment of $\beta$ PIX contains the GEF activity required for the activation of Rac1-induced membrane ruffles [24] and interacts with Abi-1 in vivo (Figure 4), the absence of the dimerisation domain in the N-terminal construct could also explain the loss of membrane dorsal ruffling.

How may the C-terminal fragment of $\beta$ PIX interfere with dorsal ruffle formation? It is possible that the C-terminal fragment of $\beta$ PIX interacts with Abi-1 in vivo in a way that does not allow co-immunoprecipitation. In this case, the $\mathrm{C}$-terminal fragment of $\beta$ PIX would also function in a dominant-negative manner by competing for binding of endogenous or overexpressed Abi-1 with endogenous $\beta$ PIX. As the C-terminal fragment of $\beta$ PIX does not possess the GEF activity, induction of membrane dorsal ruffles in response to PDGF cannot be achieved directly through the interaction between Abi- 1 and the $\beta$ PIX Cterminal fragment. Other reports where $\beta$ PIX full-length was overexpressed in fibroblasts with a $\mathrm{C}$-terminal fragment of $\beta$ PIX have shown the loss of membrane dorsal ruffles in response to PDGF, suggesting the importance of the N-terminal domain of $\beta$ PIX $[31,32]$. Furthermore, the $\mathrm{C}$-terminal fragment of $\beta$ PIX containing PxxP motifs could directly compete for the binding of Sos-1 to the SH3 domain of Abi-1 [19]. Indeed, in serum-starved fibroblasts, an interaction between Abi- 1 and Sos- 1 induces membrane dorsal ruffles in response to PDGF [19].

How might this interaction play a role in PDGF-induced actin cytoskeleton reorganisation? We propose two models. The first model implicates the interaction between Abi-1 and $\beta$ PIX as part of an alternative Ras-dependent pathway. Activation of the PDGF receptor induces a signalling cascade involving the interaction between Abi-1 and Sos-1. Sos-1 functions as a Rac-GEF downstream of Ras in a complex with the proteins Eps8, Abi-1, and PI3-K [19, $21,23]$. Within this complex, Abi-1 serves as a scaffold bridging together Eps8 and Sos-1. In an alternative route of the same pathway, Abi-1 may interact and activate the RacGEF $\beta$ PIX and thereby induces membrane dorsal ruffles. A second model of the interaction between Abi-1 and $\beta$ PIX involves a Ras-independent pathway. Rac1 can also be activated through a Ras-independent pathway involving a direct recruitment of PI-3K to activated receptor tyrosine kinases to induce Rac1-mediated cytoskeletal reorganisation $[21,39]$. The interaction between the p 85 regulatory 
subunit of PI3-K and Abi-1 has been shown to be important for Abi-1-dependent Rac1 activation and membrane dorsal ruffle formation [23]. Thus, Abi-1 may serve as a scaffold bridging PI3-K and $\beta$ PIX to allow activation of the GEF activity of $\beta$ PIX.

In summary, a novel interaction between Abi-1 and $\beta$ PIX was identified in this study, which may coordinate the formation of membrane dorsal ruffles in response to PDGF. The translation of growth signals to changes in the architecture of the cytoskeleton is a highly complex process involving different signalling pathways and multiple protein complexes. Since the whole process is highly dynamic, it is to be expected that the formation and resolution of different signalling complexes initiated by apparent redundant pathways is also highly dynamic. In this scenario, the multiple pathways initiated by growth receptors rather reflect the complexity of signalling machinery required for the coordinate regulation of the cytoskeleton than redundant parallel ways to the same outcome.

\section{Acknowledgments}

We thank Giorgio Scita (Istituto FIRC di Oncologia Molecolare, Milan, Italy) for providing the Abi-1 monoclonal antibody used for immunoprecipitation of endogenous Abi-1. We thank Trent Fowler for critical reading of the manuscript. This work was supported by Grant Nos. RU631/2-1 and RU631/2-2 to T.R.

\section{References}

1 Etienne-Manneville S, Hall A. Rho GTPases in cell biology. Nature 2002; 420:629-635.

2 Van Aelst L, D'Souza-Schorey C. Rho GTPases and signaling networks. Genes Dev 1997; 11:2295-2322.

3 Schmidt A, Hall A. Guanine nucleotide exchange factors for Rho GTPases: turning on the switch. Genes Dev 2002; 16:15871609.

4 Ridley AJ, Hall A. The small GTP-binding protein rho regulates the assembly of focal adhesions and actin stress fibers in response to growth factors. Cell 1992; 70:389-399.

5 Ridley AJ, Paterson HF, Johnston CL, Diekmann D, Hall A. The small GTP-binding protein rac regulates growth factor-induced membrane ruffling. Cell 1992; 70:401-410.

6 Nobes CD, Hall A. Rho, rac, and cdc42 GTPases regulate the assembly of multimolecular focal complexes associated with actin stress fibers, lamellipodia, and filopodia. Cell 1995; 81:53-62.

7 Stradal T, Courtney KD, Rottner K, et al. The Abl interactor proteins localize to sites of actin polymerization at the tips of lamellipodia and filopodia. Curr Biol 2001; 11:891-895.

8 Ichigotani Y, Fujii K, Hamaguchi M, Matsuda S. In search of a function for the E3B1/Abi2/Argbp1/NESH family (Review). Int J Mol Med 2002; 9:591-595.

9 Smith JM, Katz S, Mayer BJ. Activation of the Abl tyrosine kinase in vivo by Src homology 3 domains from the Src homology
2/Src homology 3 adaptor Nck. J Biol Chem 1999; 274:2795627962.

10 Fazioli F, Minichiello L, Matoska V, et al. Eps8, a substrate for the epidermal growth factor receptor kinase, enhances EGFdependent mitogenic signals. EMBO J 1993; 12:3799-3808.

11 Biesova Z, Piccoli C, Wong WT. Isolation and characterization of e3B1, an eps 8 binding protein that regulates cell growth. Oncogene 1997; 14:233-241.

12 Cowan CA, Henkemeyer M. The SH2/SH3 adaptor Grb4 transduces B-ephrin reverse signals. Nature 2001; 413:174-179.

13 Ziemnicka-Kotula D, Xu J, Gu H, et al. Identification of a candidate human spectrin Src homology 3 domain-binding protein suggests a general mechanism of association of tyrosine kinases with the spectrin-based membrane skeleton. J Biol Chem 1998; 273:13681-13692.

14 So CW, So CK, Cheung N, et al. The interaction between EEN and Abi-1, two MLL fusion partners, and synaptojanin and dynamin: implications for leukaemogenesis. Leukemia 2000; 14:594-601.

15 Mongiovi AM, Romano PR, Panni S, et al. A novel peptide-SH3 interaction. EMBO J 1999; 18:5300-5309.

16 Rogers S, Wells R, Rechsteiner M. Amino acid sequences common to rapidly degraded proteins: the PEST hypothesis. Science 1986; 234:364-368.

17 Dai Z, Pendergast AM. Abi-2, a novel SH3-containing protein interacts with the c-Abl tyrosine kinase and modulates c-Abl transforming activity. Genes Dev 1995; 9:2569-2582.

18 Wang B, Mysliwiec T, Krainc D, et al. Identification of ArgBP1, an Arg protein tyrosine kinase binding protein that is the human homologue of a CNS-specific Xenopus gene. Oncogene 1996; 12:1921-1929.

19 Scita G, Nordstrom J, Carbone R, et al. EPS8 and E3B1 transduce signals from Ras to Rac. Nature 1999; 401:290-293.

20 Plattner R, Kadlec L, DeMali KA, Kazlauskas A, Pendergast AM. c-Abl is activated by growth factors and Src family kinases and has a role in the cellular response to PDGF. Genes Dev 1999; 13:2400-2411.

21 Scita G, Tenca P, Areces LB, et al. An effector region in Eps8 is responsible for the activation of the Rac-specific GEF activity of Sos-1 and for the proper localization of the Rac-based actinpolymerizing machine. J Cell Biol 2001; 154:1031-1044.

22 Innocenti M, Tenca P, Frittoli E, et al. Mechanisms through which Sos- 1 coordinates the activation of Ras and Rac. J Cell Biol 2002; 156:125-136.

23 Innocenti M, Frittoli E, Ponzanelli I, et al. Phosphoinositide 3kinase activates Rac by entering in a complex with Eps8, Abi1, and Sos-1. J Cell Biol 2003; 160:17-23.

24 Manser E, Loo TH, Koh CG, et al. PAK kinases are directly coupled to the PIX family of nucleotide exchange factors. Mol Cell 1998; 1:183-192.

25 Bagrodia S, Taylor SJ, Jordon KA, Van Aelst L, Cerione RA. A novel regulator of p21-activated kinases. J Biol Chem 1998; 273:23633-23636.

26 Cerione RA, Zheng Y. The Dbl family of oncogenes. Curr Opin Cell Biol 1996; 8:216-222.

27 Aghazadeh B, Zhu K, Kubiseski TJ, et al. Structure and mutagenesis of the Dbl homology domain. Nat Struct Biol 1998; 5:1098-1107.

28 Zhao ZS, Manser E, Loo TH, Lim L. Coupling of PAK-inter- 
acting exchange factor PIX to GIT1 promotes focal complex disassembly. Mol Cell Biol 2000; 20:6354-6363.

29 Turner CE, Brown MC, Perrotta JA, et al. Paxillin LD4 motif binds PAK and PIX through a novel $95-\mathrm{kD}$ ankyrin repeat, ARFGAP protein: A role in cytoskeletal remodeling. J Cell Biol 1999; 145:851-863.

30 Bagrodia S, Bailey D, Lenard Z, et al. A tyrosine-phosphorylated protein that binds to an important regulatory region on the cool family of p21-activated kinase-binding proteins. J Biol Chem 1999; 274:22393-22400.

31 Kim S, Lee SH, Park D. Leucine zipper-mediated homodimerization of the p21-activated kinase-interacting factor, beta Pix. Implication for a role in cytoskeletal reorganization. J Biol Chem 2001; 276:10581-10584.

32 Koh CG, Manser E, Zhao ZS, Ng CP, Lim L. Beta1PIX, the PAK-interacting exchange factor, requires localization via a coiled-coil region to promote microvillus-like structures and membrane ruffles. J Cell Sci 2001; 114:4239-4251.

33 Ridley AJ, Schwartz MA, Burridge K, et al. Cell migration: integrating signals from front to back. Science 2003; 302:17041709.

34 Obermeier A, Ahmed S, Manser E, et al. PAK promotes morphological changes by acting upstream of Rac. EMBO J 1998; 17:4328-4339.

35 Mayer BJ. SH3 domains: complexity in moderation. J Cell Sci 2001; 114:1253-1263.

36 Fan PD, Goff SP. Abl interactor 1 binds to sos and inhibits epidermal growth factor- and v-Abl-induced activation of extracellular signal-regulated kinases. Mol Cell Biol 2000; 20:7591-7601.

37 Harlan JE, Hajduk PJ, Yoon HS, Fesik SW. Pleckstrin homology domains bind to phosphatidylinositol-4,5-bisphosphate. Nature 1994; 371:168-170.

38 Lemmon MA, Ferguson KM, Abrams CS. Pleckstrin homology domains and the cytoskeleton. FEBS Lett 2002; 513:71-76.

39 Wennstrom S, Siegbahn A, Yokote K, et al. Membrane ruffling and chemotaxis transduced by the PDGF beta-receptor require the binding site for phosphatidylinositol 3' kinase. Oncogene 1994; 9:651-660. 